\title{
Frühsportliche Giftelei
}

\section{Jann Schwarzenbach}

Dr. med., Facharzt für Allgemeinmedizin, Mitglied FMH

\author{
Klimamord und assistierter Suizid sind Themenkreise, denen sich niemand wirk- \\ lich entziehen kann. Das führt dann zu gegensätzlichen Standpunkten und ver- \\ schiedenartigen Lösungsvorschlägen.
}

Sportlichen Schrittes durchstreift der Morgenmensch Dr. Mattiniero tagtäglich die Strassen von Paradiso. Vom himmlischen Blick auf den Luganersee einmal abgesehen, wird das am Fusse des San Salvatore gelegene Betonkonglomerat seinem vielversprechenden Namen kaum noch gerecht. Die selbständige Gemeinde hat zwar eine angenehm übersichtliche Grösse, niedrige Steuern und funktionierende Institutionen. Ja, sogar ein Altersheim und ein - noch normales Postamt. Doch dann kommt halt gleich diese ganze, irrwitzige Blechlawine. Besonders schlimm ist es am Morgen, wenn die Luft eigentlich am feinsten sein sollte und der Dottore seinen Frühsport absolviert. Den damit verbundenen pulmonalen $\mathrm{CO}_{2}$-Mehrausstoss nimmt er gelassen hin: Für Ärzte und Ärztinnen geht Koronarschutz wohl immer noch vor Klimaschutz. So meint er denn, dass es viel eher die kolonnenbilden-

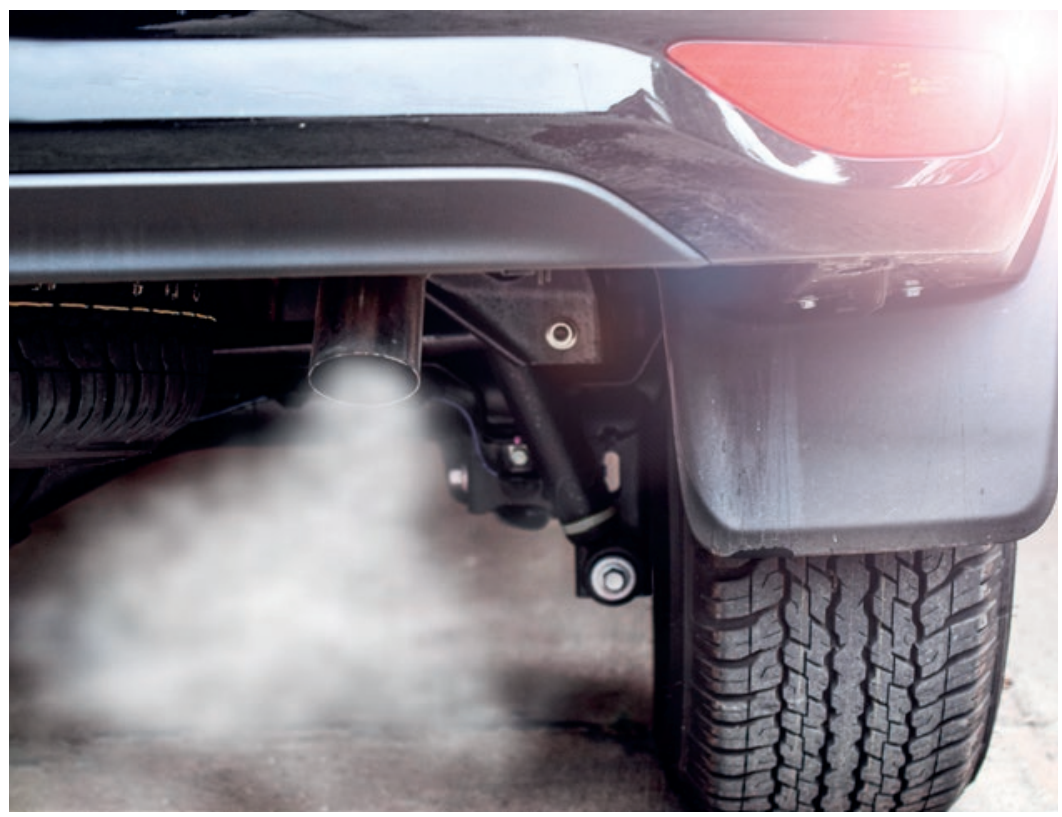

Eigentlich müssten die Jungmütter in ihren Schulkindertaxis für die künftige Gesundheit des Planeten sorgen, findet Dr. Mattiniero auf seinem Morgenspaziergang. den Jungmütter in ihren Schulkindertaxis seien, die für die zukünftige Gesundheit des Planeten sorgen müssten. Für die Insassen des nahe gelegenen Altersheims hingegen ist das eh kein Thema mehr. Verkehrsinfarkte und stinkende SUVs berühren sie kaum. Man beachtet hier vielmehr die lautstarken, schockfarbigen und ganz besonders die langgezogenen, pechschwarzen Fahrzeuge. Nicht dass man sich unbedingt davor fürchtete, dahin heimzukehren, von wo man einst gestartet war. Nein, es ist dieser künftige, bewusst zu erlebende, letzte Moment des Be-

Wie es wohl in einem Todesengel aussehen muss, nachdem er jahraus, jahrein mit dem Giftköfferchen zur Arbeit gefahren ist?

wusstseins, der alle ängstigt - nicht das Totsein also, sondern das Totwerden. Das führt immer wieder zu interdisziplinären Herausforderungen für Freundinnen und Freunde, Verwandte, Pflegepersonal, seelsorgerisch Tätige sowie Hausärztinnen und -ärzte - denn mit dem Idealbild des sanften Entschlafens ist es halt in der Praxis so eine Sache. Und da steht dann manchmal auch das Begehren im Raum, die Dinge selbst in die Hand zu nehmen. Dr. Mattiniero möchte sich einem derartigen Wunsch nicht a priori verschliessen und lobt unser Rechtssystem, das hier viel eigenverantwortliche Freiheit gewährt. Diese will er aber keinesfalls an einschlägige Institutionen übertragen. Beförderungsaufträge unterschreibe er grundsätzlich nur am nahe gelegenen Postschalter, ätzt der alte Zyniker. Es geht ihm dabei nicht um Gewissen und Moral, sondern einfach um die Frage, ob er in einer Gesellschaft leben möchte, wo «Diplomierter Einschläferungshelfer» zur gängigen Berufsbezeichnung werden könnte und der ausländischen Kundschaft anstatt Roaminggebühren Abräumungsgebühren inklusive Mehrwertsteuer belastet würden. Doch viel mehr als diese glücklicherweise spekulativen Taktlosigkeiten schreckt ihn der ganz konkrete Gedanke, 
wie es denn wohl im Innern eines solchen Todesengels einmal aussehen muss, nachdem er jeden Morgen, jahraus, jahrein mit seinem Giftköfferchen zur Arbeit gefahren ist.

\section{Institution versus kritische Reflexion}

Dr. Mattiniero giftelt also gegen eine institutionelle Selbstverständlichung und nicht gegen die assistierte Selbsttötung an sich. Er ist aber in einer Kultur aufgewachsen, wo andere Meinungen noch gerne angehört und respektiert wurden. So möchte er denn - ohne jeden Anspruch auf Vollständigkeit - eine kleine Auswahl von Denkern rund um diesen Themenkomplex zu Worte kommen lassen. Sie konnten sich zwar zu

\section{Logik der Routine anstelle individueller Ab- wägung. Dies führe zu einer Enthumanisierung der Gesellschaft.}

Lebzeiten naturgemäss noch keine Meinung zu Sterbehilfeorganisationen bilden. Trotzdem hinterliessen sie uns einige allgemeine Betrachtungen über den Suizid und zum Wesen und zur Funktion von Institutionen. Der altgriechische Lebenskünstler Epikur (341-270 v.Chr.) beispielsweise sieht bei entsprechendem Leidensdruck einen selbstgewählten Notausgang ausdrücklich vor. Arthur Schopenhauer (1788-1860) ist da hingegen völlig anderer Ansicht. Für ihn ist der Suizid keine taugliche Lösung, er sei nämlich keine Ausschaltung des Lebenswillens, sondern gerade dessen extremste Manifestation. Anders gesagt: In Wahrheit möchte der Selbstmörder gar nicht sterben, sondern äusserst gerne einfach schmerzloser leben. Diametral entgegengesetzte Meinungen gibt es auch zu unseren Institutionen. Ganz allgemein regeln und vereinheitlichen sie komplexe Aufgaben des Zusammenlebens und entlasten das Individuum damit in Bezug auf dessen persönliche Verantwortung. Für den rechtskonservativen Philosophen und Soziologen Arnold Gehlen (1904-1976), dessen beruflicher Stern schon Nazideutschland erleuchtete, galten solche normierten Strukturen und Verhaltensweisen als notwendige Kompensation für unsere - im Gegensatz $\mathrm{zu}$ anderen Lebewesen - mangelhaft ausgeprägten Instinkte. Mit anderen Worten: Um uns nicht über jeden Einzelfall den Kopf zu zerbrechen, brauche es selbständig handelnde Einrichtungen, die unser Denkorgan vom alltäglichen Krimskrams entlasten und dieses somit für edlere Aufgaben freihalten würden. Sein linksprogressiver Gegenspieler und Patenonkel der 68er-Bewegung, Theodor Adorno (1903-1969), sah gerade in diesem Outsourcing von Zuständigkeiten eine
Lähmung eigenen Denkens sowie einen Verlust von Kreativität und zwischenmenschlicher Spontaneität. Institutionen zeigten nämlich - egal für welche Werte sie anfangs eingestanden seien - eine fatale Tendenz zum Eigenleben, das sich zunehmend jeder kritischen Reflexion entziehe. Nackte Logik der Routine also anstelle individueller Abwägung. Dies führe schliesslich $\mathrm{zu}$ einer schleichenden Enthumanisierung unserer Gesellschaft.

\section{Schopenhauer als Familienplaner}

Dr. Mattiniero möchte das jetzt einfach mal so stehen lassen, um sich wieder der Umweltproblematik zuzuwenden, von der er ja anfänglich ausgegangen ist. So fragt er sich, ob die obgenannten Denker wohl dazu beitragen könnten, dem Klimamord und unserem damit verbundenen kollektiven Suizid Gegensteuer zu geben. Epikur fällt da gänzlich weg. Für diesen Apostel eines schmerzlos angenehmen Hier und Jetzt wären solche Zukunftssorgen nicht mehr als ein unnützer Stress. Arnold Gehlen würde die Sache wahrscheinlich mit Hilfe mächtiger Umwelt-Institutionen per Diktat angehen, und das wäre durchaus nicht unproblematisch: Verordnete Einsichten haben es nämlich erfahrungsgemäss schwer, in den Köpfen des Homo sapiens wirklich Fuss zu fassen. Theodor Adorno hingegen zöge wohl auch beim Klimaschutz die spontane, menschliche Kreativität und eigenverantwortliches Denken allen Institutionen vor. Doch gerade beim Denken, da hapert es eben beim breiten Volk. Da mutet ihm der gesellschaftskritische Intellektuelle allzu viel zu. Vielleicht täte hier ja wirklich

\section{Das einzig mögliche Glück des Menschen} liegt für diesen Philosophen im Totalverzicht auf alle unsere Bedürfnisse.

eine Prophetin not, so eine wie Greta Thunberg, die uns die elende Situation laut und deutlich entgegenschreit. Bei Schopenhauer gäbe es allerdings noch eine andere, weit stillere und mindestens ebenso wirksame Lösung. Das einzig mögliche Glück des Menschen liegt für diesen bärbeissigen Philosophen nämlich in der Askese, dem Totalverzicht auf alle unsere Bedürfnisse. Eine solche Maximalforderung erscheint dem lebenslustigen Dottore natürlich übertrieben. Aber eine weltweite Enthaltsamkeit auf Zeit wäre für ihn schon eine Option. Sie wäre dank der damit verbundenen Senkung der Geburtenrate sogar sehr nachhaltig.

Bildnachweis

Veerathada Khaipet | Dreamstime.com, Symobolbild 